\title{
The irradiated tumor microenvironment: role of tumor-associated macrophages in vascular recovery
}

\author{
Jeffery S. Russell ${ }^{1}$ and J. Martin Brown ${ }^{2 *}$ \\ Department of Medical Oncology, Stanford University School of Medicine, Stanford, CA, USA \\ 2 Division of Cancer and Radiation Biology, Department of Radiation Oncology, Stanford University School of Medicine, Palo Alto, CA, USA
}

\author{
Edited by: \\ Michal A. Rahat, Technion - Israel \\ Institute for Technology, Israel \\ Reviewed by: \\ Seth B. Coffelt, Netherlands Cancer \\ Institute, Netherlands \\ Lily Wu, University of California, Los \\ Angeles, USA \\ *Correspondence: \\ J. Martin Brown, Division of Cancer \\ and Radiation Biology, Department \\ of Radiation Oncology, Stanford \\ University School of Medicine, \\ 1050A Arastradero Rd., Rm A246, \\ Palo Alto, Stanford, \\ CA 94304-1334, USA \\ e-mail: mbrown@stanford.edu
}

Radiotherapy is an important modality used in the treatment of more than $50 \%$ of cancer patients in the US. However, despite sophisticated techniques for radiation delivery as well as the combination of radiation with chemotherapy, tumors can recur. Thus, any method of improving the local control of the primary tumor by radiotherapy would produce a major improvement in the curability of cancer patients. One of the challenges in the field is to understand how the tumor vasculature can regrow after radiation in order to support tumor recurrence, as it is unlikely that any of the endothelial cells within the tumor could survive the doses given in a typical radiotherapy regimen. There is now considerable evidence from both preclinical and clinical studies that the tumor vasculature can be restored following radiotherapy from an influx of circulating cells consisting primarily of bone marrow derived monocytes and macrophages. The radiation-induced influx of bone marrow derived cells (BMDCs) into tumors can be prevented through the blockade of various cytokine pathways and such strategies can inhibit tumor recurrence. However, the post-radiation interactions between surviving tumor cells, recruited immune cells, and the remaining stroma remain poorly defined. While prior studies have described the monocyte/macrophage inflammatory response within normal tissues and in the tumor microenvironment, less is known about this response with respect to a tumor after radiation therapy. The goal of this review is to summarize existing research studies to provide an understanding of how the myelomonocytic lineage may influence vascular recovery within the irradiated tumor microenvironment.

Keywords: radiation, macrophages, vasculogenesis, angiogenesis, blood vessels, tumor growth

\section{TUMOR-ASSOCIATED MACROPHAGES}

Infiltrating leukocytes are a common finding in solid tumors, first described by Virchow in 1863 and confirmed in modern studies (Wood and Gollahon, 1977; Milas et al., 1987; Balkwill and Mantovani, 2001). Tumor-associated macrophages (TAMs) are recruited to tumors and can promote tumor growth, survival, and may result in resistance to therapeutic treatments (De Palma and Lewis, 2013). As tumors mature, they acquire a heterogeneous, infiltrative population of bone marrow-derived cells (BMDCs), including a diverse array of myelomonocytic cells: neutrophils, dendritic cells, myeloid derived suppressor cells and monocytes/macrophages (Akashi et al., 2000; Nagaraj and Gabrilovich, 2010). The extent of TAM infiltration appears to correlate with a poor clinical prognosis and an increase in tumor burden (Takanami et al., 1999; Shieh et al., 2009; Toge et al., 2009).

TAMs originate as circulating monocytes recruited to tumors by cytokine gradients produced by tumor cells as well as the tumor stroma (Mantovani et al., 1992). A diverse array of cytokines and growth factors has been demonstrated to stimulate macrophage recruitment to tumors (Balkwill, 2004; Allavena et al., 2008). Initially, TAMs were felt to have anti-tumor properties; however, Mantovani et al. found that isolated macrophages from a weakly immunogenic sarcoma cell line were able to stimulate tumor cell growth in vitro (Mantovani, 1978). TAMs have now been found by many investigators to also promote tumor growth in vivo, often by producing a proangiogenic environment (Folkman, 1974; Polverini et al., 1977; Lin and Pollard, 2004).

As a simplified paradigm, macrophages are frequently considered to be polarized toward two specific phenotypes; however, it is important to realize there are many macrophage phenotypes with different specialized functions (Qian and Pollard, 2010). Classically activated macrophages (M1) are "pro-inflammatory" cells designed to protect the host from pathogenic infections. M1 macrophages are stimulated by LPS and IFN-gamma to produce IL-12, IL-6, inducible NO synthase (iNOS), and TNF-alpha (Modolell et al., 1995). These M1 populations have an enhanced ability to generate reactive oxygen species, upregulate phagocytosis, and have enhanced functionality as antigen presenting cells (Martinez et al., 2009). In contrast, M2 macrophages (alternatively activated) are considered "anti-inflammatory" as they promote tissue repair through IL-4, IL-13 and prostaglandin signaling and result in the production of IL-10 and TGF-beta (Corraliza et al., 1995; Mantovani et al., 2009). M2 populations are able to suppress cytokine production and reduce activation of $\mathrm{T}$-cells, decrease antigen presenting ability, promote angiogenesis, stimulate extracellular matrix degradation, and enhance cell survival (Murdoch et al., 2008; Lu et al., 2011). Increased arginase I (Arg I) expression is often used as a marker of the M2 phenotype 
due to changes in arginine metabolism away from $\mathrm{NO}$ generation to polyamine production (Ho and Sly, 2009). Many studies have found that the tumor microenvironment preferentially polarizes TAMs to the M2 phenotype (Gabrilovich et al., 2012; Ruffell et al., 2012).

The activation or polarization toward a particular macrophage phenotype appears to be dependent on the cytokine milieu, the presence of specific growth factors, and the level of hypoxia within the tumor microenvironment (Munder et al., 1998; Goerdt et al., 1999; Gordon, 2003; Mosser, 2003; Stout et al., 2005). However, it should be noted that macrophages can also simultaneously produce M1 as well as M2-related cytokines and that the expression is highly dependent on the tumor type, stage, and location as well as the host microenvironment. Additionally, the dynamic tumor environment may constantly shift the ratio of macrophage phenotypes depending on the current environmental conditions (Murdoch et al., 2004; Pollard, 2004). For example, an unregulated M1 population could result in a shift toward a chronic inflammatory state, while an uncontrolled M2 population could result in severe immunosuppression (Mantovani et al., 2004; Condeelis and Pollard, 2006). Furthermore, lack of M1 signals (i.e., downregulated tumor/stromal production of IL-4, IL-10, and IL-13) drive TAMs toward the M2 phenotype (Mantovani et al., 2004; Pollard, 2004; Solinas et al., 2009).
In summary, TAMs are bone marrow derived monocytic cells with unique functional subsets that are recruited to tumors by cytokine gradients and frequently differentiate into the M2 macrophage phenotype. TAM infiltration can result in an immunosuppressed environment, the promotion of proangiogenic pathways, and consequently, enhanced tumor growth and tumor cell survival.

\section{HYPOXIA AND THE PROANGIOGENIC ROLE OF TAMS}

The tumor microenvironment is often transiently or chronically in a state of low oxygen tension (Vaupel and Mayer, 2007). To survive in a hypoxic environment, tumors must establish a functional vascular network (See Figure 1). Tumors frequently adapt to hypoxia by preventing the degradation of hypoxia-induced transcription factor complexes (i.e., HIF1 and HIF2) resulting in their stabilization and subsequent transcription of genes that promote tumor survival, including proangiogenic cytokines (Giaccia et al., 2004; Keith et al., 2012). Using primary human macrophages, Fang et al. demonstrated that HIF1 and HIF2 co-regulate many hypoxia-related genes; however, by using siRNA specific knockdown studies of HIF1 and HIF2, they found that each of these genes can target certain hypoxia-associated genes independently (Fang et al., 2009).

TAMs commonly associate in necrotic, low oxygenated areas of tumors (Leek et al., 1996; Burke et al., 2003; Lewis and Murdoch,
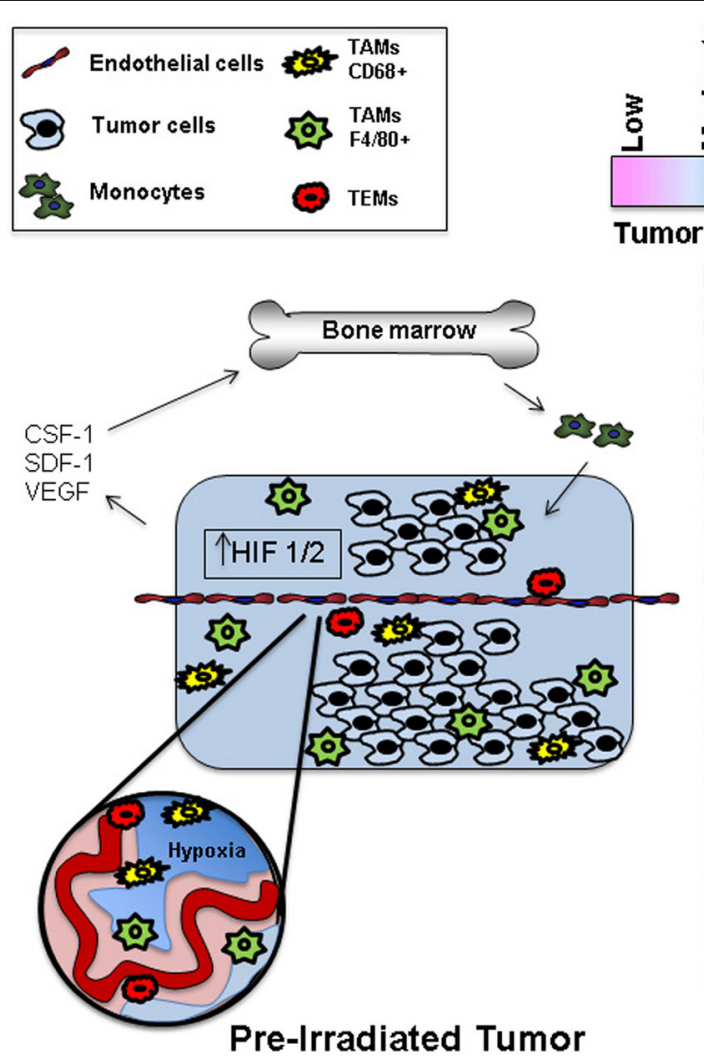

FIGURE 1 | Representation ofthe microenvironment of pre- and postirradiated tumors. The post-irradiated tumor has increased levels of hypoxia, upregulated HIF 1/2 signaling, and expression of a diverse spectrum
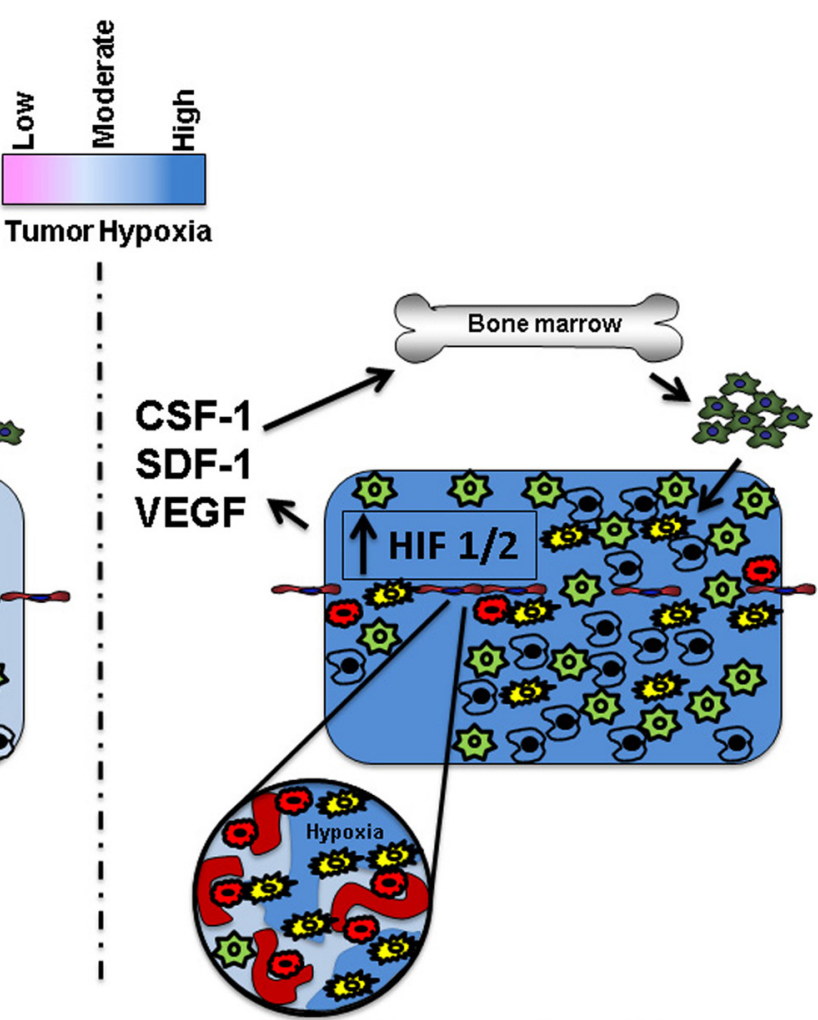

Post-Irradiated Tumor

of cytokines as well as a greater recruitment and influx of bone marrow derived TAMs. TEMs commonly associate with the vasculature, while CD68+ TAMs frequently localize to areas of severe hypoxia. 
2005). Hypoxic environments increase expression of the CXCR4 receptor on TAMs and increase the chemotactic response to its ligand, stromal cell-derived factor-1 (SDF-1/CXCL-12) (Schioppa et al., 2003). Localization of TAMs to hypoxic regions is mediated by cytokine gradients resulting from hypoxia-induced HIF1/2 stabilization (Talks et al., 2000; Murdoch et al., 2004; Jin et al., 2006; Knowles and Harris, 2007; Han et al., 2008). Cramer et al. demonstrated that HIF1 expression was required for myeloid cell motility and invasiveness (Cramer et al., 2003). Furthermore, Du et al. demonstrated that intracranial implants of HIF1-deficient glioma cells had reduced levels of infiltrating monocytes within tumors as well as reduced levels of tumor SDF-1 and MMP-9 protein expression (Du et al., 2008).

Modulation of HIF1/2 has been demonstrated to affect macrophage phenotype as well as function. However, Werno et al. demonstrated, using a macrophage lineage HIF1 knockout model, that TAM infiltration of tumors was not dependent on HIF1, but HIF1 was necessary to promote the polarization of TAMs to the M2 phenotype (Werno et al., 2010). Consistent with this, Doedens and colleagues showed that whereas hypoxia suppressed T-cell activation within tumors and resulted in tumor progression, T-cell suppression was reduced in a HIF1 macrophage lineage specific knockout model and resulted in decreased tumor growth (Doedens et al., 2010). Both HIF1 and HIF2 appear to be important regulators of the $\mathrm{M} 1$ and $\mathrm{M} 2$ polarization phenotypes. Takeda et al. reported that classical activation cytokines (IFNgamma, LPS) increased HIF1 mRNA, but strongly repressed HIF2 mRNA production, and, conversely, IL-4, the alternative activation cytokine, resulted in an increase of HIF2 mRNA (Takeda et al., 2010). Furthermore, M2-polarized macrophages demonstrated an upregulation of HIF2 mRNA. HIF2 was also found to regulate Arg I protein expression; however, in contrast, HIF1 stabilization increased iNOS expression. Deletion of HIF2 in mouse macrophages resulted in the inability to generate an appropriate inflammatory response and murine tumors implanted in the HIF2 depleted macrophage mouse model demonstrated decreased TAM infiltration as well as decreased levels of the CSFR1 and CXCR4 receptors (Imtiyaz et al., 2010). Hypoxia-induced HIF1/2 activation and the resulting differential effects on TAMs remains a complicated, highly regulated system playing a significant role in tumor progression and survival.

With respect to angiogenesis, increased macrophage infiltration in tumors is associated with a higher vascular density in breast, glioma, bladder, and esophageal tumors (Leek et al., 1996; Nishie et al., 1999; Hanada et al., 2000; Koide et al., 2004). TAMs promote tumor angiogenesis and vascularization by releasing proangiogenic cytokines such as VEGF and the matrix metalloproteinases (Lewis and Pollard, 2006). A study using breast tumor spheroids found increased VEGF levels and increased vascular connections when incubated with a macrophage population (Bingle et al., 2006). Similarly, Lewis et al. found that VEGF mRNA was upregulated in macrophages associated with human breast cancer (Lewis et al., 2000). Inhibition of VEGF and VEGFR2 by monoclonal antibodies decreased macrophage infiltration of in vivo pancreatic tumors (Dineen et al., 2008).
Interestingly, Stockmann et al. used a VEGF-A macrophage specific lineage knockout in the MMTV-PyMT breast cancer model and demonstrated similar levels of tumor-associated macrophage infiltration, decreased tumor VEGFR2 activation, and a decrease in the length of tumor blood vessels; however, overall tumor growth was actually enhanced (Stockmann et al., 2008). These results indicate differential effects of tumor-produced VEGF-A compared to macrophage-produced VEGF-A on the vascular network. Further experiments demonstrated that tumor cell death was enhanced by chemotherapy in tumor-bearing mice lacking myeloid-specific VEGF-A, suggesting that targeting the proangiogenic function of TAMs could sensitize tumors to cytotoxic therapy. Further interplay of macrophages and endothelial cells was recently demonstrated by $\mathrm{He}$ et al. who demonstrated that BM-derived hematopoietic cells incubated in vitro with immortalized endothelial cell layers resulted in the generation of M2-like macrophage colonies (He et al., 2012). Modulation of the extracellular matrix also impacts the development of vascular networks. Coussens et al. demonstrated upregulated matrix metalloproteinase-9 (MMP-9) production by bone marrow derived TAMs and increased tumor angiogenesis (Coussens et al., 2000). In a related study, Hao et al. found that BMDCs were recruited to areas of high VEGF expression, expressed elevated levels of MMP-9, and that capillary development was greatly reduced in MMP-9 knockout mice (Hao et al., 2008). Thus, the activation of macrophages within a hypoxic environment results in the release of proangiogenic cytokines and extracellular matrix modulating factors.

A subpopulation of tumor-associated macrophages/ monocytes has been identified that express the Tie 2 angiopoietin receptor and are defined as Tie2-expressing monocytes (TEMs) (De Palma et al., 2005). De Palma et al. using a suicide gene strategy showed that the selective killing of TEMs, prevented angiogenesis, and slowed tumor growth in mouse xenografts (De Palma et al., 2003). Importantly, TEM elimination did not significantly reduce the overall number of TAMs, indicating that TEMs are a small subpopulation of macrophages/monocytes. Venneri et al. demonstrated that a TEM population was present in human cancers and that these cells were responsive in vitro to chemotactic migratory stimulation by Ang2, the ligand for the Tie2 receptor (Venneri et al., 2007). Furthermore, co-injection studies of TEMs with human glioma cells resulted in more vascularized tumor xenografts in contrast to co-injection with TEM-depleted monocyte populations. Of note, TEMs associated with tumors were found to express the Tie2 receptor at elevated levels over those of circulating TEMs. Finally, tumor overexpression of Ang2, resulted in increased accumulation of TEMs within the tumor microenvironment (Coffelt et al., 2010). Hypoxia was also demonstrated to upregulate Tie2 expression in TEMs and downregulate TNF-alpha and IL-12 levels, known antiangiogenic cytokines (Murdoch et al., 2007). Hypoxia induced by vascular disrupting agents produced an increase in tumor SDF-1 expression as well as increased infiltration of CXCR4+ TEMs (Welford et al., 2011). While TAMs infiltrate the hypoxic and necrotic regions of tumors, TEMs are more frequently localized around tumor blood vessels, possess greater proangiogenic qualities, and can function as an immunosuppressive cell, similar 
to the M2 macrophage phenotype (De Palma et al., 2005; Pucci et al., 2009; Coffelt et al., 2011).

In addition to the Tie2 population, other proangiogenic TAM subsets within tumors have been identified including: CD11b+VEGFR1+ hematopoietic cells and CD11c+MCH-II+ dendritic cell precursors (Hattori et al., 2002; Yang et al., 2004; De Palma and Naldini, 2006). Similarly, CD11b+Gr-1+ myeloid cells were also found to mediate resistance to anti-VEGF therapies (Shojaei et al., 2007); however, this population may be related more toward neutrophils rather than macrophages (Shojaei and Ferrara, 2008). Movadehi et al. found that MHC II ${ }^{\text {hi }}$ and MHC $\mathrm{II}^{\text {low }}$ subsets of TAMs were associated with M1 and M2 phenotypes, respectively (Movahedi et al., 2010). Furthermore, using the chorioallantoic membrane (CAM) assay of angiogenesis, MHC II ${ }^{\text {low }}$ TAMs had a two-fold higher vessel count compared to MHC II ${ }^{\text {hi }}$ TAMs, demonstrating the enhanced proangiogenic ability of MHC II ${ }^{\text {low }}$ TAMs.

TAMs are comprised of several distinct subpopulations and are recruited to hypoxic regions of tumors via cytokine signaling gradients. In turn, they secrete growth factors to promote blood vessel formation and proteinases that remodel the tumor vascular network. Restoration of the vascular supply can result in tumor survival, proliferation, and potentially, an increased risk of metastasis.

\section{INTRINSIC RADIATION RESPONSE OF MONOCYTES/MACROPHAGES}

While previous studies found that stimulated monocytes/macrophages are innately resistant to radiotherapy, as they were demonstrated to be post-mitotic cells, Jenkins et al. reported that the activation of M2-polarized macrophages resulted in a higher cell proliferation (Hildebrandt et al., 1998; Jenkins et al., 2011). This result suggests that within the acute response to ionizing radiation, M2 macrophages may actually be more sensitive to radiation-induced DNA damage and result in cell death, in contrast to quiescent M1-polarized macrophages.

In addition to the intrinsic radiosensitivity of particular M1 or M2 TAM subsets, the influence of ionizing radiation on macrophage function may be an even more important factor in tumor survival. Early in vitro research found that radiation interferes with the recognition and degradation of antigens and results in the failure of macrophages to generate antibody responses against these targets (Donaldson et al., 1956; Nelson and Becker, 1959; Pribnow and Silverman, 1967). Similarly, Geiger et al. reported that macrophages of irradiated mice were unable to stimulate antibody production against Shigella, however, the phagocytic activity of irradiated macrophages was not impaired (Geiger and Gallily, 1974). In contrast, Lambert et al. demonstrated that in vitro radiation resulted in the priming of the macrophage cell line RAW 264.7 and upregulated MHC Class I molecules (Lambert and Paulnock, 1987). Additionally, radiation was found to augment antibody-dependent cell-mediated cytotoxicity (ADCC) in the murine macrophage cell line J774 (Duerst and Werberig, 1991). Other studies have also confirmed that radiation induces the activation of macrophages in vitro and in vivo through increased rates of phagocytosis, lysosomal enzyme production, and $\mathrm{H}_{2} \mathrm{O}_{2}$ production as well as the retained capacity to respond to cytokines (Sablonniere et al., 1983; Gallin et al., 1985; Gallin and Green, 1987; Hester and Coggin, 1989). Thus, while the antigen-presenting functions of macrophages are disrupted by radiation, their innate phagocytic function remains intact.

With respect to cytokine production, radiation-exposed macrophages have increased IL-1beta mRNA expression, upregulated TNF-alpha production, are able to potentiate nitric oxide production by interferon-gamma, and release a variety of growth factors (i.e., PDGF, IGF-1) (Sherman et al., 1991; O'Brien-Ladner et al., 1993; Iwamoto and McBride, 1994; Nemoto et al., 1995; Thornton et al., 1996; Vodovotz et al., 1999; McKinney et al., 2000). Therefore, the modulation of cytokine production by ionizing radiation may influence the macrophage polarization phenotype and function. Coates et al. found that macrophages from irradiated C57BL/6 mice demonstrated enhanced M2 activity while irradiated macrophages from $\mathrm{CBA} / \mathrm{CaJ}$ mice had increased M1 activity (Coates et al., 2008). This result suggested that ionizing radiation can induce a phenotypic polarization shift, but overall, macrophage polarization is dependent on the background genetic environment.

The above studies demonstrate that ionizing radiation directly affects macrophage function. The understanding of the intrinsic radiation response of macrophages, including disrupted antigen recognition, modulation of macrophage polarization to an immunosuppressive phenotype, and the production of proangiogenic cytokines may result in the identification of signaling pathways that could be targeted to generate a more radiosensitive subpopulation of macrophages, and ultimately, an increase in tumor responsiveness to radiation therapy.

\section{TAMS AND THE IRRADIATED MICROENVIRONMENT}

Radiosensitivity describes the in vitro response of cells to ionizing radiation, a property that depends critically on the ability of the cells to repair DNA as well as the activation of other intrinsic survival pathways. The radioresponse of tumors is defined as the in vivo change in tumor size after radiation therapy. Several components of the tumor microenvironment that can greatly affect the radioresponse of tumors are: the level of tissue oxygenation, the sensitivity of tumor endothelial cells to radiation, activation of tumor stroma to express survival factors, and immune cell infiltration of the tumor.

An influential study from the joint laboratories of Fuks and Kolesnick proposed that the radiation sensitivity of tumors to dose fractions of $10 \mathrm{~Gy}$ or more was governed by the sensitivity of tumor endothelial cells to apoptosis (Garcia-Barros et al., 2003). However, earlier data from the Suit laboratory had shown that the radiation dose to control $50 \%$ of transplanted tumors in mice did not depend on the radiation sensitivity of the tumor stroma (Budach et al., 1993). This apparent contradiction can be explained by the different assays of tumor response used-growth delay in the Fuks/Kolesnick study and tumor control (TCD50) by the Suit lab. Indeed, the dual contribution of tumor cell radiosensitivity and stromal sensitivities was demonstrated by Gerweck et al. using the growth delay assay (Ogawa et al., 2007). Several additional reports have also demonstrated that radiation results in the reduction of blood vessel density (Song et al., 1974; Timke et al., 2008; Zeng et al., 2008; Kioi et al., 2010). 
In addition to the intrinsic sensitivity of the tumor cells at the time of irradiation, the effects on the tumor stroma can also produce events that regulate tumor radioresponse. For example, the destruction of the vasculature by ionizing radiation causes hypoxic conditions which results in the activation of HIF-1, stimulation of cytokine signaling cascades, and the recruitment of macrophages and immune cells (Moeller et al., 2004; Li et al., 2007; Kioi et al., 2010). This can also occur in normal tissues as well: alveolar macrophages in the selectively irradiated mouse lung were shown to increase at 8 weeks post-treatment indicating the local organ repopulation of macrophages through tissue resident precursors or from bone marrow progenitor cells (Gross, 1977; Peel and Coggle, 1980). Similarly, Johnston et al. found that after 15 Gy of thoracic radiation, macrophages and lymphocytes were elevated within irradiated normal tissues at 16 and 24 weeks post-treatment (Johnston et al., 2004).

Milas et al. found that the tumor-associated macrophage content varied widely between in vivo tumor implants, but there was a trend toward increased macrophage content and reduced local tumor radiocurability (Milas et al., 1987). A second study confirmed that a high macrophage content in tumors was able to overcome the growth delay seen in pre-irradiated tumor beds implying the importance to TAMs for tumor angiogenesis (Milas, 1990). Similar studies have also demonstrated tumor infiltration of BMDCs after treatment with ionizing radiation (Stephens et al., 1978; Jung et al., 1990; Chen et al., 2009). Using a prostate cancer cell line in mouse xenografts, Tsai et al. demonstrated that radiation-induced TAM accumulation occurred 1-2 weeks after treatment and that irradiated TAMs expressed elevated Arg I levels suggesting an M2 phenotype (Tsai et al., 2007). Additionally, when irradiated TAMs were co-injected with tumor cells, the resulting tumors demonstrated enhanced growth rates compared to samples co-injected with unirradiated TAMs. With respect to the clinical setting, Baeten et al. found increased CD68+ macrophages in tumor biopsy samples of rectal cancer patients after radiotherapy and Kioi et al. demonstrated an increase in $\mathrm{CD} 11 \mathrm{~b}+$ myeloid cells in glioblastomas recurring after radiation (Baeten et al., 2006; Kioi et al., 2010).

Ahn et al. demonstrated that radiation treatment of MT1A2 mouse mammary tumors results in an influx of CD11b+ cells expressing high levels in MMP-9 in either irradiated tumors or tumors grown in a pre-irradiated tumor bed (Ahn and Brown, 2008). Additionally, the expression of MMP-9 by CD11b+ myelomonocytes was necessary for vascular restoration and tumor growth in irradiated tissues. Finally, selective depletion of CD11b+ cells by a monoclonal antibody inhibited tumor growth in pre-irradiated tissues (Ahn et al., 2010). Taken together, these data demonstrate that TAMs promote tumor growth and stimulates early tumor regrowth through improved blood vessel formation.

The influx of TAMs after radiation appears to be the result of increased levels of the transcription factor HIF-1, secondary to increased tumor hypoxia after irradiation. Using an dual inhibitor of both HIF-1 and HIF-2, Kioi et al. found a decrease in the number of radiation-induced BMDC infiltration (mostly $\mathrm{CD} 11 \mathrm{~b}+$ cells) in an orthotopic mouse xenograft model of human glioblastoma (Kioi et al., 2010). Similarly, treatment of mice with irradiated tumors using carrageenan, to deplete systemic monocytes/macrophages, also resulted in decreased tumor infiltration of $\mathrm{CD} 11 \mathrm{~b}+$ cells after radiation treatment. Further, ionizing radiation induced elevated levels of the downstream HIF-1 target, SDF-1, within U251 tumor xenografts. Blocking the interaction of SDF-1 and its receptor CXCR4, by using the CXCR4 inhibitor AMD3100 or a CXCR4 neutralizing antibody, resulted in decreased tumor perfusion and an enhanced radioresponse of the glioma xenograft model. Interestingly, Kozin et al. also showed an increase in CD11b + myeloid cells in irradiated tumors and demonstrated that whole body radiation (depleting the bone marrow compartment) combined with the local irradiation of a tumor site resulted in improved local tumor control compared to local radiation alone (Kozin et al., 2010). Additionally, an infusion of myeloid progenitor cells improved tumor regrowth after local radiation. Similar to the study by Kioi et al. SDF-1 was also found to be upregulated in irradiated tumor tissues and blocking the SDF-1/CXCR4 interaction with AMD3100 inhibited tumor re-growth after radiation. Both studies found that Tie2+ BMDCs were significantly increased in tumors after local radiation and that these cells, while localized to the vasculature, were not incorporated into tumor vessel walls.

Chiang et al. found that CD68+ TAMs accumulate in hypoxic regions of certain tumors, but this is dependent on the tumor type as well as the local microenvironment (Chiang et al., 2012). However, after radiation therapy, CD11b+ myeloid cells were distributed into distinct spatial locations: CD68+ TAMs were found in areas of central hypoxia, while F4/80+ TAMs were found on the edge of hypoxic regions adjacent to necrotic regions. They proposed that radiation therapy may activate specific factors to localize or retain CD68+ TAMs into anoxic or hypoxic regions. Finally, they determined that the radiation-activated CD68+ TAMs expressed Arg I, indicating a polarization toward the M2 phenotype of macrophage, and that TAM recruitment was dependent on SDF-1. An additional study has indicated that tumors implanted into pre-irradiated fields grow slower than in unirradiated control tissues (i.e., the "tumor bed effect") and demonstrate an aggregation of CD68+ TAMs in hypoxic regions (Chen et al., 2011). Furthermore, when BMDCs were injected systemically into mice with tumors grown in a pre-irradiated field, they incorporated specifically into the tumor vasculature of the low blood vessel density regions.

In addition to the SDF-1/CXCR4 pathway enhanced by radiation-induced tumor hypoxia, the CSF-1/CSF1R signaling complex has also been recently implicated in recruitment of myeloid cells to growing tumors and in promoting the radiation-induced monocytic infiltration of tumors. Dorsch et al. demonstrated that transfection of the human CSF-1 gene into a synergetic mouse model resulted in increased TAM infiltration of the tumor (Dorsch et al., 1993). Another study determined that the CSF-1 ligand could stimulate monocytes to produce VEGF and form microtubule structures in vitro (Eubank et al., 2003). Using a small molecule inhibitor to the receptor of CSF-1, Priceman et al. found that the CSF-1/CSF1R pathway was necessary for the recruitment of TAMs, promoted tumor progression, and the release of proangiogenic cytokines (Priceman et al., 2010). 
Recently, $\mathrm{Xu}$ et al. demonstrated that radiation increased TAM accumulation in tumors, upregulated in vivo tumor expression of CSF-1 and interestingly, in irradiated prostate cancer patients, found that serum levels of CSF-1 were also increased (Xu et al., 2013). A selective inhibitor of the CSF-1 receptor combined with radiation therapy suppressed tumor growth compared to radiation alone. They proposed that the mechanism for the increased CSF-1 expression in tumors was by radiation-induced DNA damage resulting in the activation and translocation of the ABL kinase into the cell nucleus, binding to the CSF-1 gene promoter, and the enhancement of CSF-1 gene transcription.

\section{CONCLUSIONS}

Following tumor irradiation, DNA damage, cell death, and increased tumor hypoxia promotes the production of VEGF, SDF-1, and CSF-1 resulting in the recruitment, infiltration, and retention of monocytes/macrophages within tumors. The recruited heterogeneous populations of TAMs release proangiogenic cytokines and metalloproteinases to promote blood vessel formation within tumors. The level of hypoxia appears to distribute particular TAM subgroups to specific regions of the tumor. While the TEM subset is frequently localized to the perivascular niche, other subpopulations of TAMs are divided across necrotic, peri-necrotic, and low oxygen tension regions. Additionally, M2 macrophage polarization appears to be the dominant phenotype within hypoxic tumors.

Radiation is a unique therapy modality as it causes DNA damage and enhances tumor hypoxia, but only within a targeted region. Radiation-induced recruitment of TAMs appears to occur in a similar manner as that caused by tumor hypoxia, is partially dependent on the SDF-1/CXCR4 and CSF-1/CSFR signaling pathways, and promotes polarization toward the M2 phenotype. Thus, the accumulation of radiation-induced TAMs within a tumor may result in the increased production of proangiogenic cytokines, the recovery of the vascular network, and consequently, tumor regrowth.

\section{REFERENCES}

Ahn, G. O., and Brown, J. M. (2008). Matrix metalloproteinase-9 is required for tumor vasculogenesis but not for angiogenesis: role of bone marrow-derived myelomonocytic cells. Cancer Cell 13, 193-205. doi: 10.1016/j.ccr. 2007.11.032

Ahn, G. O., Tseng, D., Liao, C. H., Dorie, M. J., Czechowicz, A., and Brown, J. M. (2010). Inhibition of Mac-1 (CD11b/CD18) enhances tumor response to radiation by reducing myeloid cell recruitment. Proc. Natl. Acad. Sci. U.S.A. 107, 8363-8368. doi: 10.1073/pnas.0911378107

Akashi, K., Traver, D., Miyamoto, T., and Weissman, I. L. (2000). A clonogenic common myeloid progenitor that gives rise to all myeloid

While the generalized process of TAM recruitment has been identified, many unanswered questions and challenges remain. First, the heterogeneous population of TAMs needs to be clearly identified both by phenotypic markers and function. Which markers clearly define the subpopulations of TAMs? Are the radiation-induced TAM populations different from the tumor resident TAMs? Furthermore, does TAM infiltration of irradiated tumors change over time (i.e., an acute response and/or a chronic response)? What are the functions of the specific TAM subgroups (i.e., cytokine release, extracellular matrix remodeling, or immunosuppression)? Second, several cytokine-related signaling pathways have been implicated in the recruitment of TAMs to irradiated tumor sites; however, much more research is needed. For example, what are the specific intracellular and extracellular signaling pathways driving TAM recruitment and distribution within a tumor? And, does radiation merely enhance hypoxic signaling or does it generate its own unique signaling network? Thus, the radiation-induced signaling pathways driving TAM recruitment, distribution, and function remain to be fully elucidated. Thirdly, retrospective clinical data suggests that increased macrophage infiltration of tumors is often a poor prognostic feature. Could the subtype of TAM infiltration into tumors be used as a more specific prognostic tool? Would it be possible to stratify patients based on the subtype of TAM infiltration (pre or post-radiation) to certain risk groups or even select for certain treatment strategies? Additional studies are needed to correlate clinical outcomes with the biological data in order to answer these questions.

Finally, evidence supports that TAMs promote tumor growth and survival. By understanding which TAM subsets are most beneficial to the tumor and by defining the intra- and extracellular pathways, novel therapies can be developed to disrupt TAM recruitment and function. Therefore, ablation of TAM infiltration within tumors may be a unique strategy to enhance the effectiveness of radiation therapy by decreasing angiogenic signaling, disrupting vascular recovery, reducing local tumor recurrence rates, and decreasing the risk of invasion and metastasis.

Rev. Cancer 4, 540-550. doi: $10.1038 / \mathrm{nrcl} 388$

lineages. Nature 404, 193-197. doi: 10.1038/35004599

Allavena, P., Sica, A., Solinas, G., Porta, C., and Mantovani, A. (2008). The inflammatory micro-environment in tumor progression: the role of tumor-associated macrophages. Crit. Rev. Oncol. Hematol. 66, 1-9. doi: 10.1016/j.critrevonc.2007. 07.004

Baeten, C. I., Castermans, K., Lammering, G., Hillen, F., Wouters, B. G., Hillen, H. F., et al. (2006). Effects of radiotherapy and chemotherapy on angiogenesis and leukocyte infiltration in rectal cancer. Int. J. Radiat. Oncol. Biol. Phys. 66, 1219-1227. doi: 10.1016/j.ijrobp. 2006.07.1362

Balkwill, F. (2004). Cancer and the chemokine network. Nat.
Balkwill, F., and Mantovani, A. (2001). Inflammation and cancer: back to Virchow? Lancet 357, 539-545. doi: 10.1016/S0140-6736(00)04046-0

Bingle, L., Lewis, C. E., Corke, K. P., Reed, M. W., and Brown, N. J. (2006). Macrophages promote angiogenesis in human breast tumour spheroids in vivo. Br. J. Cancer 94, 101-107. doi: 10.1038/sj.bjc.6602901

Budach, W., Taghian, A., Freeman, J., Gioioso, D., and Suit, H. D. (1993). Impact of stromal sensitivity on radiation response of tumors. J. Natl. Cancer Inst. 85, 988-993.

Burke, B., Giannoudis, A., Corke, K. P., Gill, D., Wells, M., ZieglerHeitbrock, L., et al. (2003). Hypoxia-induced gene expression in human macrophages: implications for ischemic tissues and hypoxia-regulated gene therapy. Am. J. Pathol. 163, 1233-1243. doi: 10.1016/S0002-9440(10)63483-9

Chen, F. H., Chiang, C. S., Wang, C. C., Fu, S. Y., Tsai, C. S., Jung, S. M., et al. (2011). Vasculatures in tumors growing from preirradiated tissues: formed by vasculogenesis and resistant to radiation and antiangiogenic therapy. Int J. Radiat. Oncol. Biol. Phys. 80, 1512-1521. doi: 10.1016/j.ijrobp. 2011.02.055

Chen, F. H., Chiang, C. S., Wang, C. C., Tsai, C. S., Jung, S. M., Lee, C. C., et al. (2009). Radiotherapy decreases vascular density and causes hypoxia with macrophage aggregation in TRAMP-C1 prostate tumors. Clin. 
Cancer Res. 15, 1721-1729. doi: 10.1158/1078-0432.CCR-08-1471

Chiang, C. S., Fu, S. Y., Wang, S. C., Yu, C. F., Chen, F. H., Lin, C. M., et al. (2012). Irradiation promotes an $\mathrm{m} 2$ macrophage phenotype in tumor hypoxia. Front. Oncol. 2:89. doi: 10.3389/fonc.2012.00089

Coates, P. J., Rundle, J. K., Lorimore, S. A., and Wright, E. G. (2008). Indirect macrophage responses to ionizing radiation: implications for genotype-dependent bystander signaling. Cancer Res. 68, 450-456. doi: 10.1158/0008-5472.CAN-07-3050

Coffelt, S. B., Chen, Y. Y., Muthana, M., Welford, A. F., Tal, A. O., Scholz, A., et al. (2011). Angiopoietin 2 stimulates TIE2-expressing monocytes to suppress $\mathrm{T}$ cell activation and to promote regulatory $\mathrm{T}$ cell expansion. J. Immunol. 186, 4183-4190. doi: 10.4049/jimmunol.1002802

Coffelt, S. B., Tal, A. O., Scholz, A., De Palma, M., Patel, S., Urbich, C., et al. (2010). Angiopoietin-2 regulates gene expression in TIE2-expressing monocytes and augments their inherent proangiogenic functions. Cancer Res. 70, 5270-5280. doi: 10.1158/0008-5472.CAN-10-0012

Condeelis, J., and Pollard, J. W. (2006). Macrophages: obligate partners for tumor cell migration, invasion, and metastasis. Cell 124, 263-266. doi: 10.1016/j.cell.2006.01.007

Corraliza, I. M., Soler, G., Eichmann, K., and Modolell, M. (1995). Arginase induction by suppressors of nitric oxide synthesis (IL-4, IL-10 and PGE2) in murine bone-marrow-derived macrophages. Biochem. Biophys. Res. Commun. 206, 667-673. doi: 10.1006/bbrc.1995.1094

Coussens, L. M., Tinkle, C. L., Hanahan, D., and Werb, Z. (2000). MMP-9 supplied by bone marrowderived cells contributes to skin carcinogenesis. Cell 103, 481-490.

Cramer, T., Yamanishi, Y., Clausen, B. E., Forster, I., Pawlinski, R., Mackman, N., et al. (2003). HIFlalpha is essential for myeloid cellmediated inflammation. Cell 112, 645-657.

De Palma, M., and Lewis, C. E. (2013). Macrophage regulation of tumor responses to anticancer therapies. Cancer Cell 23, 277-286. doi: 10.1016/j.ccr.2013.02.013

De Palma, M., and Naldini, L. (2006). Role of haematopoietic cells and endothelial progenitors in tumour angiogenesis. Biochim. Biophys. Acta 1766, 159-166. doi: 10.1016/j.bbcan.2006.06.003

De Palma, M., Venneri, M. A., Galli, R., Sergi Sergi, L., Politi, L. S.,
Sampaolesi, M., et al. (2005). Tie2 identifies a hematopoietic lineage of proangiogenic monocytes required for tumor vessel formation and a mesenchymal population of pericyte progenitors. Cancer Cell 8, 211-226. doi: 10.1016/j.ccr.2005.08.002

De Palma, M., Venneri, M. A., Roca, C., and Naldini, L. (2003). Targeting exogenous genes to tumor angiogenesis by transplantation of genetically modified hematopoietic stem cells. Nat. Med. 9, 789-795. doi: 10.1038/nm871

Dineen, S. P., Lynn, K. D., Holloway, S. E., Miller, A. F., Sullivan, J. P., Shames, D. S., et al. (2008). Vascular endothelial growth factor receptor 2 mediates macrophage infiltration into orthotopic pancreatic tumors in mice. Cancer Res. 68, 4340-4346. doi: 10.1158/00085472.CAN-07-6705

Doedens, A. L., Stockmann, C., Rubinstein, M. P., Liao, D., Zhang, N., DeNardo, D. G., et al. (2010). Macrophage expression of hypoxia-inducible factor-1 alpha suppresses T-cell function and promotes tumor progression. Cancer Res. 70, 7465-7475. doi: 10.1158/0008-5472.CAN-10-1439

Donaldson, D. M., Marcus, S., Gyi, K. K., and Perkins, E. H. (1956). The influence of immunization and total body $\mathrm{x}$-irradiation on intracellular digestion by peritoneal phagocytes. J. Immunol. 76, 192-199.

Dorsch, M., Hock, H., Kunzendorf, U., Diamantstein, T., and Blankenstein, T. (1993). Macrophage colonystimulating factor gene transfer into tumor cells induces macrophage infiltration but not tumor suppression. Eur. J. Immunol. 23, 186-190. doi: 10.1002/eji.1830230129

Du, R., Lu, K. V., Petritsch, C., Liu, P., Ganss, R., Passegue, E., et al. (2008). HIFlalpha induces the recruitment of bone marrowderived vascular modulatory cells to regulate tumor angiogenesis and invasion. Cancer Cell 13, 206-220. doi: 10.1016/j.ccr.2008.01.034

Duerst, R., and Werberig, K. (1991). Cells of the J774 macrophage cell line are primed for antibody-dependent cellmediated cytotoxicity following exposure to gamma-irradiation. Cell. Immunol. 136, 361-372.

Eubank, T. D., Galloway, M., Montague, C. M., Waldman, W. J., and Marsh, C. B. (2003). M-CSF induces vascular endothelial growth factor production and angiogenic activity from human monocytes. J. Immunol. 171, 2637-2643.
Fang, H. Y., Hughes, R., Murdoch, C., Coffelt, S. B., Biswas, S. K., Harris, A. L., et al. (2009). Hypoxia-inducible factors 1 and 2 are important transcriptional effectors in primary macrophages experiencing hypoxia. Blood 114 844-859. doi: 10.1182/blood-200812-195941

Folkman, J. (1974). Tumor angiogenesis. Adv. Cancer Res. 19, 331-358.

Gabrilovich, D. I., Ostrand-Rosenberg, S., and Bronte, V. (2012). Coordinated regulation of myeloid cells by tumours. Nat. Rev. Immunol. 12, 253-268. doi: 10.1038/nri3175

Gallin, E. K., and Green, S. W. (1987). Exposure to gamma-irradiation increases phorbol myristate acetateinduced $\mathrm{H} 2 \mathrm{O} 2$ production in human macrophages. Blood 70, 694-701.

Gallin, E. K., Green, S. W., and Sheehy, P. A. (1985). Enhanced activity of the macrophage-like cell line J774.1 following exposure to gamma radiation. J. Leukoc. Biol. 38, 369-381.

Garcia-Barros, M., Paris, F., CordonCardo, C., Lyden, D., Rafii, S., Haimovitz-Friedman, A., et al. (2003). Tumor response to radiotherapy regulated by endothelial cell apoptosis. Science 300, 1155-1159. doi: 10.1126/science. 1082504

Geiger, B., and Gallily, R. (1974). Effect of X-irradiation on various functions of murine macrophages. Clin. Exp. Immunol. 16, 643-655.

Giaccia, A. J., Simon, M. C., and Johnson, R. (2004). The biology of hypoxia: the role of oxygen sensing in development, normal function, and disease. Genes Dev. 18, 2183-2194. doi 10.1101/gad.1243304

Goerdt, S., Politz, O., Schledzewski, K., Birk, R., Gratchev, A., Guillot, P., et al. (1999). Alternative versus classical activation of macrophages. Pathobiology 67, 222-226. doi: $10.1159 / 000028096$

Gordon, S. (2003). Alternative activation of macrophages. Nat. Rev. Immunol. 3, 23-35. doi: $10.1038 /$ nri978

Gross, N. J. (1977). Alveolar macrophage number: an index of the effect of radiation on the lungs. Radiat. Res. 72, 325-332.

Han, Z. B., Ren, H., Zhao, H., Chi, Y., Chen, K., Zhou, B., et al. (2008). Hypoxia-inducible factor (HIF)-1 alpha directly enhances the transcriptional activity of stem cell factor (SCF) in response to hypoxia and epidermal growth factor (EGF). Carcinogenesis 29, 1853-1861. doi: 10.1093/carcin/bgn066
Hanada, T., Nakagawa, M., Emoto, A., Nomura, T., Nasu, N., and Nomura, Y. (2000). Prognostic value of tumor-associated macrophage count in human bladder cancer. Int J. Urol. 7, 263-269.

Hao, Q., Liu, J., Pappu, R., Su, H., Rola, R., Gabriel, R. A., et al. (2008). Contribution of bone marrow-derived cells associated with brain angiogenesis is primarily through leukocytes and macrophages. Arterioscler. Thromb. Vasc. Biol. 28, 2151-2157. doi: 10.1161/ATVBAHA.108.176297

Hattori, K., Heissig, B., Wu, Y., Dias, S., Tejada, R., Ferris, B., et al. (2002). Placental growth factor reconstitutes hematopoiesis by recruiting VEGFR1(+) stem cells from bone-marrow microenvironment. Nat. Med. 8, 841-849. doi: 10.1038/nm740

He, H., Xu, J., Warren, C. M., Duan, D., Li, X., Wu, L., et al. (2012). Endothelial cells provide an instructive niche for the differentiation and functional polarization of M2-like macrophages. Blood 120, 3152-3162. doi: 10.1182/blood-2012-04-422758

Hester, R. B., and Coggin, J. H. Jr. (1989). Tumorigenic sublethal whole-body X-irradiation of RFM mice enhances cell-mediated cytotoxicity while transiently depressing T- and B-lymphocytes. Mol. Biother. 1, 244-249.

Hildebrandt, G., Seed, M. P., Freemantle, C. N., Alam, C. A., Colville-Nash, P. R., and Trott, K. R. (1998). Mechanisms of the antiinflammatory activity of low-dose radiation therapy. Int. J. Radiat. Biol. 74, 367-378.

Ho, V. W., and Sly, L. M. (2009). Derivation and characterization of murine alternatively activated (M2) macrophages. Methods Mol. Biol. 531, 173-185. doi: 10.1007/978-159745-396-7_12

Imtiyaz, H. Z., Williams, E. P., Hickey, M. M., Patel, S. A., Durham, A. C., Yuan, L. J., et al. (2010). Hypoxia-inducible factor 2alpha regulates macrophage function in mouse models of acute and tumor inflammation. J. Clin. Invest. 120, 2699-2714. doi: 10.1172/JCI39506

Iwamoto, K. S., and McBride, W. H. (1994). Production of 13hydroxyoctadecadienoic acid and tumor necrosis factor-alpha by murine peritoneal macrophages in response to irradiation. Radiat. Res. 139, 103-108.

Jenkins, S. J., Ruckerl, D., Cook, P. C. Jones, L. H., Finkelman, F. D., van Rooijen, N., et al. (2011). Local 
macrophage proliferation, rather than recruitment from the blood, is a signature of TH2 inflammation. Science 332, 1284-1288. doi: 10.1126/science. 1204351

Jin, D. K., Shido, K., Kopp, H. G., Petit, I., Shmelkov, S. V., Young, L. M., et al. (2006). Cytokine-mediated deployment of SDF-1 induces revascularization through recruitment of CXCR4+ hemangiocytes. Nat. Med. 12, 557-567. doi: 10.1038/nm1400

Johnston, C. J., Williams, J. P., Elder, A., Hernady, E., and Finkelstein, J. N. (2004). Inflammatory cell recruitment following thoracic irradiation. Exp. Lung Res. 30, 369-382. doi: $10.1080 / 01902140490438915$

Jung, H., Kruger, H. J., Brammer, I., Zywietz, F., and Beck-Bornholdt, H. P. (1990). Cell population kinetics of the rhabdomyosarcoma $\mathrm{R} 1 \mathrm{H}$ of the rat after single doses of X-rays. Int. J. Radiat. Biol. 57, 567-589.

Keith, B., Johnson, R. S., and Simon, M. C. (2012). HIFlalpha and HIF2alpha: sibling rivalry in hypoxic tumour growth and progression. Nat. Rev. Cancer 12, 9-22. doi: $10.1038 / \mathrm{nrc} 3183$

Kioi, M., Vogel, H., Schultz, G., Hoffman, R. M., Harsh, G. R., and Brown, J. M. (2010). Inhibition of vasculogenesis, but not angiogenesis, prevents the recurrence of glioblastoma after irradiation in mice. J. Clin. Invest. 120, 694-705. doi: 10.1172/JCI40283

Knowles, H. J., and Harris, A. L. (2007). Macrophages and the hypoxic tumour microenvironment. Front. Biosci. 12, 4298-4314.

Koide, N., Nishio, A., Sato, T., Sugiyama, A., and Miyagawa, S. (2004). Significance of macrophage chemoattractant protein-1 expression and macrophage infiltration in squamous cell carcinoma of the esophagus. Am. J. Gastroenterol. 99, 1667-1674. doi: 10.1111/j.1572-0241.2004.30733.x

Kozin, S. V., Kamoun, W. S., Huang, Y., Dawson, M. R., Jain, R. K., and Duda, D. G. (2010). Recruitment of myeloid but not endothelial precursor cells facilitates tumor regrowth after local irradiation. Cancer Res. 70, 5679-5685. doi: 10.1158/00085472.CAN-09-4446

Lambert, L. E., and Paulnock, D. M. (1987). Modulation of macrophage function by gamma-irradiation. Acquisition of the primed cell intermediate stage of the macrophage tumoricidal activation pathway. J. Immunol. 139, 2834-2841.

Leek, R. D., Lewis, C. E., Whitehouse, R., Greenall, M., Clarke, J., and Harris, A. L. (1996). Association of macrophage infiltration with angiogenesis and prognosis in invasive breast carcinoma. Cancer Res. 56, 4625-4629.

Lewis, C., and Murdoch, C. (2005). Macrophage responses to hypoxia: implications for tumor progression and anti-cancer therapies. Am. J. Pathol. 167, 627-635. doi: 10.1016/S0002-9440(10)62038-X

Lewis, C. E., and Pollard, J. W. (2006). Distinct role of macrophages in different tumor microenvironments. Cancer Res. 66, 605-612. doi: 10.1158/0008-5472.CAN-05-4005

Lewis, J. S., Landers, R. J., Underwood, J. C., Harris, A. L., and Lewis, C. E. (2000). Expression of vascular endothelial growth factor by macrophages is up-regulated in poorly vascularized areas of breast carcinomas. J. Pathol. 192, 150-158. doi: 10.1002/1096-9896 (2000)9999:9999<::AID-PATH687 $>3.0 . \mathrm{CO} ; 2-\mathrm{G}$

Li, F., Sonveaux, P., Rabbani, Z. N., Liu, S., Yan, B., Huang, Q., et al. (2007). Regulation of HIF-1alpha stability through Snitrosylation. Mol. Cell 26, 63-74. doi: 10.1016/j.molcel.2007.02.024

Lin, E. Y., and Pollard, J. W. (2004). Role of infiltrated leucocytes in tumour growth and spread. $B r$. J. Cancer 90, 2053-2058. doi: 10.1038/sj.bjc.6601705

Lu, T., Ramakrishnan, R., Altiok, S., Youn, J. I., Cheng, P., Celis, E., et al. (2011). Tumor-infiltrating myeloid cells induce tumor cell resistance to cytotoxic $\mathrm{T}$ cells in mice. J. Clin. Invest. 121, 4015-4029. doi: 10.1172/JCI45862

Mantovani, A. (1978). Effects on in vitro tumor growth of murine macrophages isolated from sarcoma lines differing in immunogenicity and metastasizing capacity. Int. J. Cancer 22, 741-746.

Mantovani, A., Allavena, P., and Sica, A. (2004). Tumour-associated macrophages as a prototypic type II polarised phagocyte population: role in tumour progression. Eur. J. Cancer 40, 1660-1667. doi: 10.1016/j.ejca.2004.03.016

Mantovani, A., Bottazzi, B., Colotta, F., Sozzani, S., and Ruco, L. (1992). The origin and function of tumor-associated macrophages. Immunol. Today 13, 265-270. doi: 10.1016/0167-5699 (92) $90008-\mathrm{U}$

Mantovani, A., Sica, A., Allavena, P., Garlanda, C., and Locati, M. (2009). Tumor-associated macrophages and the related myeloid-derived suppressor cells as a paradigm of the diversity of macrophage activation.
Hum. Immunol. 70, 325-330. doi: 10.1016/j.humimm.2009.02.008

Martinez, F. O., Helming, L., and Gordon, S. (2009). Alternative activation of macrophages: an immunologic functional perspective. Annu. Rev. Immunol. 27, 451-483. doi: 10.1146/ annurev.immunol.021908.132532

McKinney, L. C., Aquilla, E. M., Coffin, D., Wink, D. A., and Vodovotz, Y. (2000). Ionizing radiation potentiates the induction of nitric oxide synthase by interferon-gamma and/or lipopolysaccharide in murine macrophage cell lines. Role of tumor necrosis factor-alpha. Ann. N.Y. Acad. Sci. 899, 61-68.

Milas, L. (1990). Tumor bed effect in murine tumors: relationship to tumor take and tumor macrophage content. Radiat. Res. 123, 232-236.

Milas, L., Wike, J., Hunter, N., Volpe, J., and Basic, I. (1987). Macrophage content of murine sarcomas and carcinomas: associations with tumor growth parameters and tumor radiocurability. Cancer Res. 47, 1069-1075.

Modolell, M., Corraliza, I. M., Link, F., Soler, G., and Eichmann, K. (1995). Reciprocal regulation of the nitric oxide synthase/arginase balance in mouse bone marrowderived macrophages by TH1 and $\mathrm{TH} 2$ cytokines. Eur. J. Immunol. 25, 1101-1104. doi: 10.1002/eji.1830250436

Moeller, B. J., Cao, Y., Li, C. Y., and Dewhirst, M. W. (2004). Radiation activates HIF-1 to regulate vascular radiosensitivity in tumors: role of reoxygenation, free radicals, and stress granules. Cancer Cell 5, 429-441.

Mosser, D. M. (2003). The many faces of macrophage activation. J. Leukoc. Biol. 73, 209-212.

Movahedi, K., Laoui, D., Gysemans, C., Baeten, M., Stange, G., Van den Bossche, J., et al. (2010). Different tumor microenvironments contain functionally distinct subsets of macrophages derived from Ly6C(high) monocytes. Cancer Res. 70, 5728-5739. doi: 10.1158/0008-5472.CAN-09-4672

Munder, M., Eichmann, K., and Modolell, M. (1998). Alternative metabolic states in murine macrophages reflected by the nitric oxide synthase/arginase balance: competitive regulation by $\mathrm{CD} 4+\mathrm{T}$ cells correlates with Th1/Th2 phenotype. J. Immunol. 160, 5347-5354.

Murdoch, C., Giannoudis, A., and Lewis, C. E. (2004). Mechanisms regulating the recruitment of macrophages into hypoxic areas of tumors and other ischemic tissues. Blood 104, 2224-2234. doi: 10.1182/blood-2004-03-1109

Murdoch, C., Muthana, M., Coffelt, S. B., and Lewis, C. E. (2008). The role of myeloid cells in the promotion of tumour angiogenesis. Nat. Rev. Cancer 8, 618-631. doi: 10.1038/nrc2444

Murdoch, C., Tazzyman, S., Webster, S. and Lewis, C. E. (2007). Expression of Tie- 2 by human monocytes and their responses to angiopoietin-2. J. Immunol. 178, 7405-7411.

Nagaraj, S., and Gabrilovich, D. I. (2010). Myeloid-derived suppressor cells in human cancer. Cancer J. 16, 348-353. doi: 10.1097/PPO.0b013e3181eb3358

Nelson, E. L., and Becker, J. R. (1959). The effect of whole-body $\mathrm{x}$ irradiation on the bactericidal activity of phagocytic cells. I. Survival of Pseudomonas aeruginosa within phagocytes from peritoneal exudates of mice. J. Infect. Dis. 104, 13-19.

Nemoto, K., Ishihara, H., Tanaka, I., Suzuki, G., Tsuneoka, K., Yoshida, K., et al. (1995). Expression of IL1 beta mRNA in mice after whole body X-irradiation. J. Radiat. Res. 36, 125-133.

Nishie, A., Ono, M., Shono, T., Fukushi, J., Otsubo, M., Onoue, H., et al. (1999). Macrophage infiltration and heme oxygenase-1 expression correlate with angiogenesis in human gliomas. Clin. Cancer Res. 5 , 1107-1113.

O'Brien-Ladner, A., Nelson, M. E., Kimler, B. F., and Wesselius, L. J. (1993). Release of interleukin-1 by human alveolar macrophages after in vitro irradiation. Radiat. Res. 136, 37-41.

Ogawa, K., Boucher, Y., Kashiwagi, S., Fukumura, D., Chen, D., and Gerweck, L. E. (2007). Influence of tumor cell and stroma sensitivity on tumor response to radiation. Cancer Res. 67, 4016-4021. doi: 10.1158 0008-5472.CAN-06-4498

Peel, D. M., and Coggle, J. E. (1980). The effect of $\mathrm{X}$ irradiation on alveolar macrophages in mice. Radiat. Res. 81, 10-19.

Pollard, J. W. (2004). Tumoureducated macrophages promote tumour progression and metastasis. Nat. Rev. Cancer 4, 71-78. doi: 10.1038/nrc1256

Polverini, P. J., Cotran, P. S., Gimbrone, M. A. Jr., and Unanue, E. R. (1977) Activated macrophages induce vascular proliferation. Nature 269, 804-806. 
Pribnow, J. F., and Silverman, M. S. (1967). Studies on the radiosensitive phase of the primary antibody response in rabbits. I. The role of the macrophage. J. Immunol. 98, 225-229.

Priceman, S. J., Sung, J. L., Shaposhnik, Z., Burton, J. B., Torres-Collado, A. X., Moughon, D. L., et al. (2010). Targeting distinct tumor-infiltrating myeloid cells by inhibiting CSF-1 receptor: combating tumor evasion of antiangiogenic therapy. Blood 115, 1461-1471. doi: 10.1182/ blood-2009-08-237412

Pucci, F., Venneri, M. A., Biziato, D., Nonis, A., Moi, D., Sica, A., et al. (2009). A distinguishing gene signature shared by tumor-infiltrating Tie2-expressing monocytes, blood "resident" monocytes, and embryonic macrophages suggests common functions and developmental relationships. Blood 114, 901-914. doi: 10.1182/blood2009-01-200931

Qian, B. Z., and Pollard, J. W. (2010). Macrophage diversity enhances tumor progression and metastasis. Cell 141, 39-51. doi: 10.1016/j.cell.2010.03.014

Ruffell, B., Affara, N. I., and Coussens, L. M. (2012). Differential macrophage programming in the tumor microenvironment. Trends Immunol. 33, 119-126. doi: 10.1016/j.it.2011.12.001

Sablonniere, B., Nicolas, J., Neveux, Y., and Drouet, J. (1983). Effect of whole-body irradiation on phagocytic activity of rat alveolar macrophages. Int. J. Radiat. Biol. Relat. Stud. Phys. Chem. Med. 44, 575-584.

Schioppa, T., Uranchimeg, B., Saccani, A., Biswas, S. K., Doni, A., Rapisarda, A., et al. (2003). Regulation of the chemokine receptor CXCR4 by hypoxia. J. Exp. Med. 198, 1391-1402. doi: 10.1084/jem.20030267

Sherman, M. L., Datta, R., Hallahan, D. E., Weichselbaum, R. R., and Kufe, D. W. (1991). Regulation of tumor necrosis factor gene expression by ionizing radiation in human myeloid leukemia cells and peripheral blood monocytes. J. Clin. Invest. 87, 1794-1797. doi: 10.1172/JCI115199

Shieh, Y. S., Hung, Y. J., Hsieh, C. B., Chen, J. S., Chou, K. C., and Liu, S. Y. (2009). Tumorassociated macrophage correlated with angiogenesis and progression of mucoepidermoid carcinoma of salivary glands. Ann. Surg. Oncol. 16, 751-760. doi: 10.1245/s10434008-0259-6
Shojaei, F., and Ferrara, N. (2008). Refractoriness to antivascular endothelial growth factor treatment: role of myeloid cells. Cancer Res. 68, 5501-5504. doi: 10.1158/0008-5472.CAN-08-0925

Shojaei, F., Wu, X., Malik, A. K., Zhong, C., Baldwin, M. E., Schanz, S., et al. (2007). Tumor refractoriness to anti-VEGF treatment is mediated by $\mathrm{CD} 11 \mathrm{~b}+\mathrm{Gr} 1+$ myeloid cells. Nat. Biotechnol. 25, 911-920. doi: 10.1038/nbt1323

Solinas, G., Germano, G., Mantovani, A., and Allavena, P. (2009). Tumor-associated macrophages (TAM) as major players of the cancer-related inflammation. J. Leukoc. Biol. 86, 1065-1073. doi: 10.1189/jlb.0609385

Song, C. W., Sung, J. H., Clement, J. J., and Levitt, S. H. (1974). Vascular changes in neuroblastoma of mice following $\mathrm{x}$-irradiation. Cancer Res. 34, 2344-2350.

Stephens, T. C., Currie, G. A., and Peacock, J. H. (1978). Repopulation of gamma-irradiated Lewis lung carcinoma by malignant cells and host macrophage progenitors. $\mathrm{Br}$. J. Cancer 38, 573-582.

Stockmann, C., Doedens, A., Weidemann, A., Zhang, N., Takeda, N., Greenberg, J. I., et al. (2008). Deletion of vascular endothelial growth factor in myeloid cells accelerates tumorigenesis. Nature 456, 814-818. doi: $10.1038 /$ nature 07445

Stout, R. D., Jiang, C., Matta, B., Tietzel, I., Watkins, S. K., and Suttles, J. (2005). Macrophages sequentially change their functional phenotype in response to changes in microenvironmental influences. J. Immunol. $175,342-349$.

Takanami, I., Takeuchi, K., and Kodaira, S. (1999). Tumorassociated macrophage infiltration in pulmonary adenocarcinoma: association with angiogenesis and poor prognosis. Oncology 57, 138-142. doi: 10.1159/000012021

Takeda, N., O’Dea, E. L., Doedens, A., Kim, J. W., Weidemann, A., Stockmann, C., et al. (2010). Differential activation and antagonistic function of HIF-\{alpha\} isoforms in macrophages are essential for NO homeostasis. Genes Dev. 24, 491-501. doi: 10.1101/gad.1881410

Talks, K. L., Turley, H., Gatter, K. C., Maxwell, P. H., Pugh, C. W., Ratcliffe, P. J., et al. (2000). The expression and distribution of the hypoxia-inducible factors HIFlalpha and HIF-2alpha in normal human tissues, cancers, and tumorassociated macrophages. Am. J. Pathol. 157, 411-421.
Thornton, S. C., Walsh, B. J., Bennett, S., Robbins, J. M., Foulcher, E., Morgan, G. W., et al. (1996) Both in vitro and in vivo irradiation are associated with induction of macrophage-derived fibroblast growth factors. Clin. Exp. Immunol. 103, 67-73.

Timke, C., Zieher, H., Roth, A., Hauser, K., Lipson, K. E., Weber, K. J., et al. (2008). Combination of vascular endothelial growth factor receptor/platelet-derived growth factor receptor inhibition markedly improves radiation tumor therapy. Clin. Cancer Res. 14, 2210-2219. doi 10.1158/1078-0432.CCR-07-1893

Toge, H., Inagaki, T., Kojimoto, Y., Shinka, T., and Hara, I. (2009). Angiogenesis in renal cell carcinoma: the role of tumor-associated macrophages. Int. J. Urol. 16, 801-807. doi: 10.1111/j.1442-2042.2009.02377.x

Tsai, C. S., Chen, F. H., Wang, C. C., Huang, H. L., Jung, S. M., Wu, C. J., et al. (2007). Macrophages from irradiated tumors express higher levels of iNOS, arginase-I and COX-2, and promote tumor growth. Int. J. Radiat. Oncol. Biol. Phys. 68, 499-507. doi: 10.1016/j.ijrobp.2007.01.041

Vaupel, P., and Mayer, A. (2007). Hypoxia in cancer: significance and impact on clinical outcome. Cancer Metastasis Rev. 26, 225-239. doi 10.1007/s10555-007-9055-1

Venneri, M. A., De Palma, M., Ponzoni, M., Pucci, F., Scielzo, C., Zonari, E., et al. (2007). Identification of proangiogenic TIE2-expressing monocytes (TEMs) in human peripheral blood and cancer. Blood 109, 5276-5285. doi: 10.1182/blood-2006-10-053504

Vodovotz, Y., Coffin, D., DeLuca, A. M., McKinney, L., Cook, J. A., Wink, D., et al. (1999). Induction of nitric oxide production in infiltrating leukocytes following in vivo irradiation of tumor-bearing mice. Radiat. Oncol. Investig. 7, 86-97. doi: 10.1002/(SICI) 1520 6823(1999)7:2<86::AID-ROI4>3. $0 . \mathrm{CO} ; 2-\mathrm{L}$

Welford, A. F., Biziato, D., Coffelt, S. B., Nucera, S., Fisher, M., Pucci, F., et al. (2011). TIE2-expressing macrophages limit the therapeutic efficacy of the vascular-disrupting agent combretastatin A4 phosphate in mice. J. Clin. Invest. 121, 1969-1973. doi: 10.1172/JCI44562

Werno, C., Menrad, H., Weigert, A., Dehne, N., Goerdt, S., Schledzewski, K., et al. (2010). Knockout of HIF-lalpha in tumor-associated macrophages enhances M2 polarization and attenuates their pro-angiogenic responses. Carcinogenesis 31, 1863-1872. doi: 10.1093/carcin/ bgq088

Wood, G. W., and Gollahon, K. A. (1977). Detection and quantitation of macrophage infiltration into primary human tumors with the use of cell-surface markers. J. Natl. Cancer Inst. 59, 1081-1087.

Xu, J., Escamilla, J., Mok, S., David, J., Priceman, S. J., West, B. L., et al. (2013). Abrogating the protumorigenic influences of tumor-infiltrating myeloid cells byCSF1R signaling blockade improves the efficacy of radiotherapy in prostatecancer. Cancer Res. 73, 2782-2794. doi: 10.1158/00085472.CAN-12-3981

Yang, L., DeBusk, L. M., Fukuda, K., Fingleton, B., Green-Jarvis, B., Shyr, Y., et al. (2004). Expansion of myeloid immune suppressor $\mathrm{Gr}+\mathrm{CD} 11 \mathrm{~b}+$ cells in tumor-bearing host directly promotes tumor angiogenesis. Cancer Cell 6, 409-421. doi: 10.1016/j.ccr.2004.08.031

Zeng, L., Ou, G., Itasaka, S., Harada, H., Xie, X., Shibuya, K., et al. (2008). TS-1 enhances the effect of radiotherapy by suppressing radiation-induced hypoxiainducible factor-1 activation and inducing endothelial cell apoptosis. Cancer Sci. 99, 2327-2335. doi: $\quad 10.1111 /$ j.1349-7006.2008. 00943.x

Conflict of Interest Statement: The authors declare that the research was conducted in the absence of any commercial or financial relationships that could be construed as a potential conflict of interest.

Received: 27 March 2013; accepted: 11 June 2013; published online: 17 July 2013.

Citation: Russell JS and Brown JM (2013) The irradiated tumor microenvironment: role of tumor-associated macrophages in vascular recovery. Front. Physiol. 4:157. doi: 10.3389/fphys. 2013.00157

This article was submitted to Frontiers in Vascular Physiology, a specialty of Frontiers in Physiology.

Copyright () 2013 Russell and Brown. This is an open-access article distributed under the terms of the Creative Commons Attribution License, which permits use, distribution and reproduction in other forums, provided the original authors and source are credited and subject to any copyright notices concerning any third-party graphics etc. 